\title{
Platelet-Rich Blood Derivatives for Stem Cell-Based Tissue Engineering and Regeneration
}

\author{
Elham A. Masoudi ${ }^{1,2}$ • João Ribas ${ }^{1,2,3}$ - Gaurav Kaushik ${ }^{1,2}$ • Jeroen Leijten ${ }^{1,2,4}$. \\ Ali Khademhosseini ${ }^{1,2,5,6,7}$
}

Published online: 13 February 2016

(C) Springer International Publishing AG 2016

\begin{abstract}
Platelet-rich blood derivatives have been widely used in different fields of medicine and stem cell-based tissue engineering. They represent natural cocktails of autologous growth factors, which could provide an alternative for recombinant protein-based approaches. Platelet-rich blood derivatives, such as platelet-rich plasma, have consistently shown to potentiate stem cell proliferation, migration, and differentiation. Here, we review the spectrum of platelet-rich blood derivatives, discuss their current applications in tissue engineering and regenerative
\end{abstract}

This article is part of the Topical Collection on Stem Cells and Nanotechnologies

Elham Masoudi and João Ribas contributed equally to this work.

Ali Khademhosseini

alik@bwh.harvard.edu

1 Department of Medicine, Biomaterials Innovation Research Center, Brigham and Women's Hospital, Harvard Medical School, Cambridge, MA 02139, USA

2 Harvard-MIT Division of Health Sciences and Technology, Massachusetts Institute of Technology, Cambridge, MA 02139, USA

3 Doctoral Program in Experimental Biology and Biomedicine, Center for Neuroscience and Cell Biology, Institute for Interdisciplinary Research, University of Coimbra, 3030-789 Coimbra, Portugal

4 Department of Developmental BioEngineering, MIRA Institute for Biomedical Technology and Technical Medicine, University of Twente, 7500AE Enschede, The Netherlands

5 Wyss Institute for Biologically Inspired Engineering, Harvard University, Boston, MA 02115, USA

6 Department of Bioindustrial Technologies, College of Animal Bioscience and Technology, Konkuk University, Hwayang-dong, Gwangjin-gu Seoul 143-701, Republic of Korea

7 Department of Physics, King Abdulaziz University, Jeddah 21569, Saudi Arabia medicine, reflect on their effect on stem cells, and highlight current translational challenges.

Keywords Blood derivatives · Platelet-rich plasma $\cdot$ Stem cells $\cdot$ Cell proliferation $\cdot$ Stem cell differentiation .

Autologous growth factors

\section{Introduction}

Tissue engineering traditionally stimulates cells using a single bioactive agent with key regenerative functions [1]. In contrast, natural tissue regeneration relies on a cocktail of signaling molecules and growth factors. During natural wound healing, activated platelets concentrate in the wound area and secrete a plethora of factors that play an instrumental role in coordinating wound healing.

Using a single growth factor to steer tissue regeneration represents an oversimplified and inefficient stimulus. This is generally overcome by providing supraphysiological quantities of the growth factor of choice, which comes at the price of adverse effects [2]. In consequence, a rapidly growing number of studies have explored the efficacy of supplementing stem cell-based tissue engineering approaches with natural growth factor cocktails such as platelet concentrates. This has paved the way for improvements in (stem) cell function including cell growth, viability, proliferation, differentiation, and overall regenerative potential [3]. Platelet concentrates are therefore becoming widely used in medicine. Moreover, their translation is being fueled by their availability, cost-effectiveness, extensive range of applications, and autologous nature. Indeed, several clinical uses of platelet concentrates have been reported in the fields of dermatology, orthopedics, dentistry, and ophthalmology [4]. 
Here, we review the spectrum of platelet-rich blood derivatives, discuss their current applications in tissue engineering and regenerative medicine, reflect on their effect on stem cells, and highlight current translational challenges.

\section{Platelet-Rich Blood Derivatives}

Activation of platelet triggers degranulation and subsequent release of trophic factors that influence wound healing, tissue repair, angiogenesis, and stem cell behavior. There are two types of granules inside the platelets: "alpha" and "dense" granules [5]. Alpha granules affect wound healing through several types of growth factors including the following: platelet-derived growth factor (PDGF), epithelial growth factor (EGF), vascular endothelial growth factor (VEGF), endothelial cell growth factor (ECGF), fibroblast growth factor (FGF), transforming growth factor beta (TGF $\beta$ ), and insulin-like growth factor (IGF). In general, these factors chemotactically recruit and activate stem cells as well as induce their mitogenesis and differentiation. In contrast, dense granules promote tissue regeneration by secreting mediators such as serotonin and histamine, which increase vessels permeability and tissue perfusion [6]. Over the years, several platelet-rich blood derivative formulations have been explored. Most notably, they have received a substantial amount of attention in tissue regeneration studies aimed at the healing of injured soft and hard tissues [7].

\section{Platelet-Rich Plasma}

Platelet-based blood derivative platelet-rich plasma (PRP) has been introduced several decades ago [8••]. PRP is prepared via a two-step centrifugation preparation of a blood sample, which was cured with an anticoagulant. During the first step of centrifugation, three layers are demarcated: plasma on top, erythrocytes on the bottom, and a middle layer that contains platelets and leukocyte. After discarding the erythrocytes, the remainder is centrifuged for a second time to ensure proper plasma separation. PRP is subsequently obtained by discarding the plasma (Fig. 1a) [9]. This process concentrates the platelet by approximately fourfold to sevenfold as compared to untreated whole blood [11].

By varying the isolation protocol, distinct mixtures can be obtained. For example, different centrifugation settings (speed, time) can alter the platelet concentration. In one such approach, Amabel et al. explored how centrifugal and temperature variables influenced the final product. Depletion of erythrocytes and white blood cells with minimal loss of platelets was considered a superior outcome. The most optimal results were obtained while centrifuging at $300 \mathrm{~g}$ for $5 \mathrm{~min}$ at $12{ }^{\circ} \mathrm{C}$ or $240 \mathrm{~g}$ for $8 \mathrm{~min}$ at $16{ }^{\circ} \mathrm{C}$ for the first spin and $700 \mathrm{~g}$ for $17 \mathrm{~min}$ at $12{ }^{\circ} \mathrm{C}$ for the second spin [8••]. In general, longer centrifugal periods slightly increased the platelet yield and decreased the concentrations of white blood cells in the upper layer. Therefore, centrifugation parameters can be used to control the amount of white blood cells in PRP [12]. Temperature proved essential to control platelet activation; low temperatures delayed platelet activation and prolonged their viability [9].

Despite its advantages, the preparation of PRP is based on several artisanal handling steps. This results in a relatively high batch-to-batch variability [13]. It could be speculated that this lack of standardization might explain some of the conflicting clinical results [14].

\section{Platelet-Rich Fibrin}

Several attempts have been made to develop new, easy-to-use platelet-derived products. This has led to the generation of platelet-rich fibrin (PRF), which is a single stage centrifuged product that does not require the addition of various chemicals. In particular, blood is centrifuged immediately after drawing to prevent coagulation. Subsequently, the middle layer is separated from the two other layers (Fig. 1b) [15]. Centrifugation is usually performed at $700 \mathrm{~g}$ for $12 \mathrm{~min}$ to obtain standard PRF (S-PRF) or at $200 \mathrm{~g}$ for $14 \mathrm{~min}$ to obtain activated PRF (APRF). Ghanaati et al. reported that speed and timing did not affect the concentrations of monocytes and stem cells, but did affect those of platelets and neutrophils. As a result, A-PRF contains more platelets, most were found at the distal layer of PRF membrane, and S-PRF includes more neutrophils [16]. This type of white blood has the potential to enhance angiogenesis by expressing the enzyme matrix metalloproteinase 9 . Therefore, the inclusion of neutrophils in PRF could be considered if angiogenesis is of interest [17].

PRF can release high quantities of multiple growth factors including TGF $\beta-1$, PDGF, and VEGF [10]. The main difference between PRF and PRP resides within their respective fibrin architectures. In PRF, this network gradually builds up during centrifugation and in the absence of anticoagulant agents. This results in a dense fibrin structure and, in PRF, acts as a network in which platelets and leukocytes are entrapped during centrifugation (Fig. 1c). This reservoir property of the fibrin network enhances the gradual release of growth factors and other mediators, resulting in prolonged maintenance and stimulation of stem cells by PRF [18]. Indeed, the release patterns of growth factor such as TGF $\beta$ and PDGF are different between PRP and PRF. In PRP, the release of TGF $\beta$ and PDGF clearly decreased after the first day, while PRF was demonstrated to release significant amounts of TGF $\beta$ and PDGF for up to 2 weeks [19]. Ehrenfest et al. corroborated this distinction in release profiles for leukocytederived VEGF in PRF versus PRP [20]. Together, these studies suggested that PRF membranes might be able to release a higher quantity of growth factors for a prolonged period of time [21].

In addition to standard formulations, PRF can also be obtained in an injectable form (I-PRF) by compressing PRF membranes between metallic sheets. Advantageously, this 
a

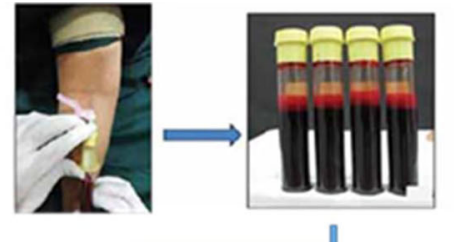

First spin
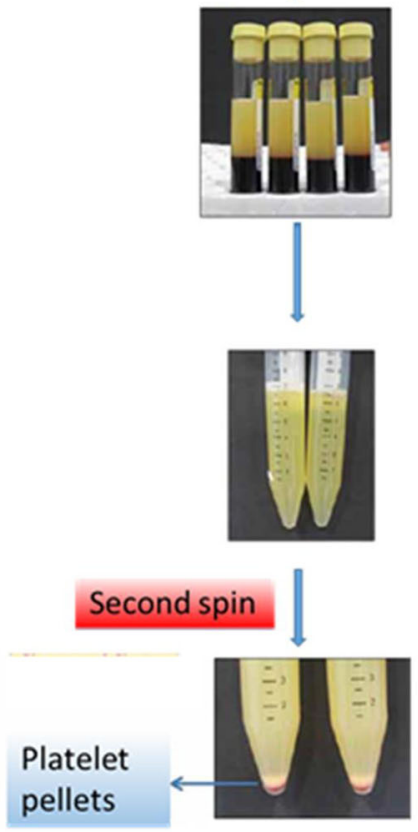

Collection of whole blood samples in anticoagulated tubes

Transfer of the buffy coat (upper layer) to empty tubes b

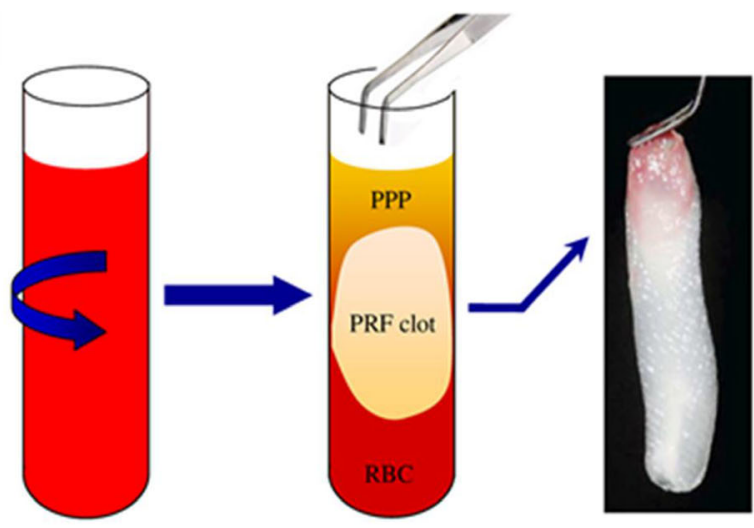

C

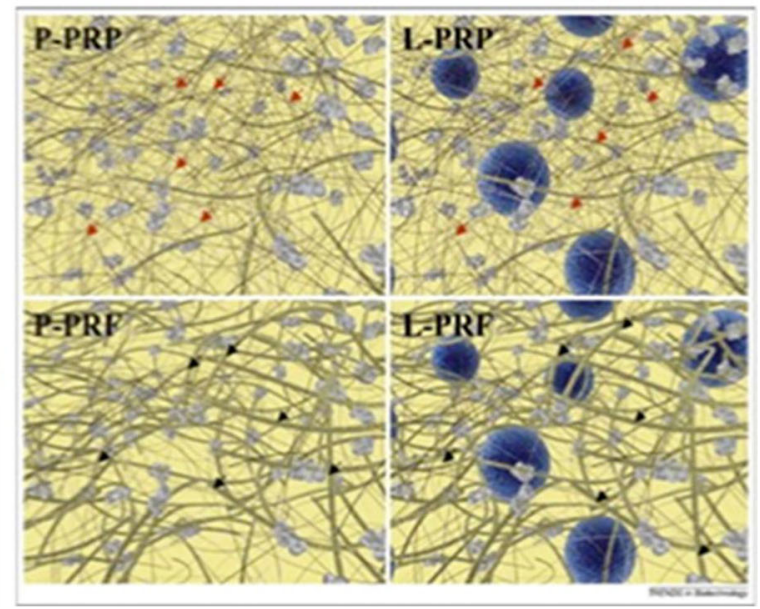

Fig. 1 Preparation protocols of (a) PRP and (b) PRF. c Schematic depiction of leucocyte-free platelet-rich plasma (P-PRP) and plateletrich fibrin (P-PRF), leucocytes PRP (L-PRP), and leucocyte- and platelet-rich plasma. $L-P R F$ leucocyte- and platelet-rich fibrin. Fibrin

injectable material can coagulate immediately postinjection to form a biomaterial as well as be combined with any biomaterial of choice for non-covalent incorporation [22].

\section{Leukocytes-Based Subclassification of PRP and PRF}

Besides platelets, leukocytes contribute to chemical composition of PRP and PRF by secreting molecules such as interleukin 1 beta (IL1 $\beta$ ), IL4, IL6, and tumor necrosis factor alpha $(\mathrm{TNF} \alpha)$, which affect wound inflammation, vascularization, and regeneration [23]. As a result, PRP and PRF have been classified into two main groups based on whether they contain or lack leukocytes. While leukocytes are present in traditional PRP and PRF (named L-PRP and L-PRF), plasmapheresis of these platelet-rich blood derivatives results in leukocyte-free 'pure' PRP and PRF (P-PRP and P-PRF).

As inflammation is one of the fundamental stages of wound healing, leukocytes can be considered as interesting cell source for both the initiation and regulation of the tissue regeneration cascade. Furthermore, they can control excess inflammation by timely release of anti-inflammatory cytokines architecture is thicker and more robust in PRF (black arrows) than PRP (red arrows). Figure 1a reprinted from Dhurat and Sukesh [9]. Figures 1b, c reprinted from Dohan Ehrenfest et al. [10]

such as IL-4, IL-10, and IL-13 [13]. Indeed, leukocytes provide an immune regulatory role and release of large amounts of VEGF and other cytokines. Regardless, the most commonly used platelet-rich blood derivatives rely on leukocyte limited formulations (L-PRP and L-PRF) [24].

\section{Growth Factor Release of PRP and PRF}

In addition to the composition of PRP and PRF, their delivery method plays a pivotal role on their ability to affect (stem) cells as well as on their clinical outcome. A hydrogel system utilizing alginate carriers for PRP growth factor release revealed that beads or capsules had different release profiles of PDGF-AB, TGF $\beta-1$, and IGF-1 [25]. Alginate beads demonstrated higher release of TGF $\beta-1$, whereas capsules favored PDGF-AB release; alginate capsules showed higher levels of SaOS-2 cell proliferation as compared to beads and controls. Utilizing the same cell line, Celotti and colleagues [26] used a neutralizing TGF $\beta$ antibody, which reduced cell proliferation. Furthermore, long-term controlled release of PRP and PRF can be obtained. Lyophilized PRP powder incorporated in 
electrospun scaffolds achieved up to 35 days release of proteins in culture and stimulated cell proliferation [5]. Furthermore, complementing PRP with a heparin-conjugated fibrin biomaterial allowed for sustained release of FGF-2, PDGF-BB, and VEGF [27]. In a mice wound closure model, this combination enabled faster wound closure and regeneration, underlining a potential role of PRP in treatment of chronic skin wounds. The ability to control the compositional release of PRP and PRF as well as its time-window can thus improve (stem) cell-based tissue engineering approaches.

\section{Effects of Platelet-Rich Plasma in Tissue Engineering}

PRP and PRF have shown promising results in various (stem) cell-based tissue engineering and regenerative medicine applications but have generated divisive results. Currently, there are over a dozen ongoing large clinical trials studying the regenerative potential of PRP, which would help elucidate the potential benefits and pitfalls of this blood derivative [4]. This section of the review aims to discuss the advances in our understanding PRP's effect on cell proliferation, differentiation, growth factor release, inflammation, and chemotaxis.

\section{Cell Proliferation}

PRP has consistently been shown to increase cell proliferation in all explored cell types. This includes differentiated cells such as osteoblast-like cells and chondrocytes $[25,26$, 28-34], periodontal ligament cells [31, 35, 36], tendon cells $[37,38]$, preadipocytes, and endothelial cells $[33,39]$ as well as multipotent cells such as mesenchymal stem cells $[40 \bullet \bullet$, 41-43] and adipose-derived stem cells [5, 44-47].

PRP mediates this increase in proliferation in a dosedependent manner [29, 33, 35, 37, 38]. Osteoblasts were shown to increase proliferation with PRP [33], although one study showed platelet poor plasma $(50 \%)$ to have a greater enhancement of cell proliferation [31]. In alveolar bone cells, higher concentrations of PRP suppressed proliferation, whereas low concentrations (1-5\%) stimulated proliferation [30]. Regardless, PRP exerts a stimulatory effect on the proliferation of stem cells such as MSCs (Fig. 2a), exhibiting also dose dependency [40••, 41-45]. Murphy and colleagues have shown that PRP derived from human umbilical cord blood had higher proliferation rates in MSCs as compared to normal blood [42]. Small concentrations $(0.1 \%)$ of umbilical cord PRP had the maximum increase in proliferation compared to different combinations of recombinant growth factors. Although the stimulation of (stem) cell proliferation could be a multifactorial event due to the numerous distinct proliferation stimulating molecules, Stat $3 / \mathrm{p} 27^{\text {Kip } 1}$ promotion of cell cycle progression has been identified as a potential mechanism [38]. Moreover, PRP can also indirectly stimulate proliferation by enhancing (stem) cell adhesion, highlighting the use of PRP for increased (stem) cell adherence in biomaterials [45]. Besides proliferation, PRP can contribute to an increase in cell expansion rates by reducing cell death. In particular, PRP supplementation reduced Bcl-2 expression and apoptosis [46].

Different animal models have corroborated the capabilities and limitations of PRP usage for cell proliferation. In a diabetic rat femur fracture model (noncritical), PRP supplementation at the fracture site increased cell proliferation, torque to failure, and torsional rigidity [49]. In another study, PRP released from gelatin gels implanted in a bone defect promoted bone regeneration (Fig. 2b). However, in a long bone defect model, PRP combined with a collagen scaffold did not exert differential effects in bone volume, mineral density, and mechanical rigidity [50]. These potentially conflicting reports might point toward the dire consequences of different growth factor compositions between distinct patients, thus emphasizing the irregular nature of PRP composition and its clinical implications [51].

Together, these reports mainly indicated PRP to have a stimulative effect on cellular proliferation in different tissues. Nevertheless, further efforts are required to understand how different preparations of PRP, individual-to-individual variation, and specific composition of PRP contribute to its effects. Moreover, little remains known on the proliferative effects and acting mechanisms of PRF on (stem) cells.

\section{Cell Differentiation}

PRP can have a potent effect on the differentiation of a variety of stem cells. PRP releasate has shown a particular affinity for stimulating the differentiation of stem cells into skeletal cell types such as cartilage, bone, blood vessel, and tendon [47, 52-54].

Periodontal ligament cells as well as osteoblast-like cell lines SaOS-2 and HOS showed increase in alkaline phosphatase (ALP) activity and increased the differentiation into mineralized tissue formation cells upon exposure to PRP [25, 34-36]. Moreover, these cell lines rapidly increased the expression of the osteogenic differentiation markers such as osteopontin (OPN), osteoprotegerin, and runt-related transcription factor 2 (RUNX2) [34]. In adipose-derived stem cells, PRP was found, at later stages (21 days), to promote osteogenic differentiation by increasing ALP, OPN, osteocalcin (OCN), and RUNX2 in a dose-dependent manner [47]. Interestingly, PRP exposure not only increased the osteogeneic potential of stem cells but also reduced their adipogenic differentiation efficacy (Fig. 2c) [40••]. PRP is hypothesized to exert its osteogenic stimulation through synergistic effects with BMP2, BMP4, BMP6, and BMP7 [55]. Indeed, BMPs have a stronger effect on osteoblast differentiation than PRP but demonstrated augmented effects when 
a

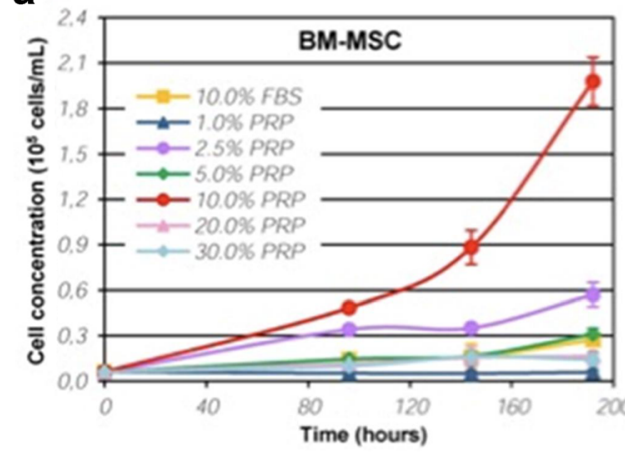

b

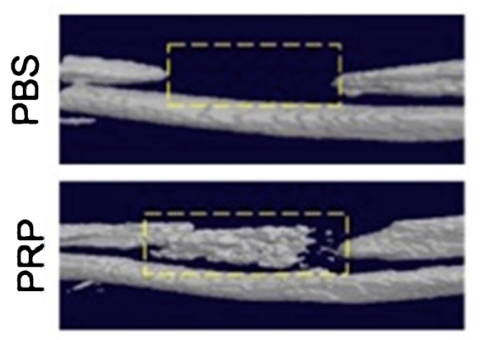

Fig. 2 Effects of PRP on stem cell proliferation, differentiation potential and bone healing. a Effect of different PRP concentrations on the proliferation of bone-marrow-derived MSCs [41]. b Three-dimensional micro-CT of a bone defect implanted with hydrogels containing either PBS or PRP [48]. c Differentiation media supplemented with PRP, bone

supplemented with PRP [29]. This makes PRP a suitable synergistic agent to steer stem cell osteogenesis for bone tissue engineering applications.

Besides osteogenesis, PRP's effect on chondrogenesis of stem cells has been well-characterized $[32,56]$. PRP supplementation to expanding chondrogenic (progenitor) cells allows for prolonged maintenance of chondrogenic potential. Moreover, addition of PRP to chondrogenic media increased the chondrogenic potential of MSCs [57]. Incorporation of PRP in polyglycolic acid-hyaluronan scaffolds increased the expression of chondrogenic markers collagen II and IX, aggrecan, and cartilage oligomeric matrix protein [58]. Furthermore, platelet lysate supplementation affected MSCs encapsulated within dextran-tyramine by chemotactically attracting them as well as by improving their subsequent attachment and chondrogenic differentiation efficacy [54]. The effects of PRF on chondrogenesis and osteogenesis have remained largely unstudied. Moreover, the contribution of PRP and PRF on the differentiation of stem cells into different tissues has not been systematically investigated and has therefore remained largely unknown.

\section{Angiogenesis}

PRP is also known to stimulate angiogenesis, which is an essential part of tissue regeneration and stem cell recruitment. It increases migration and tube formation in HUVECs [39]. In a coculture of endothelial progenitor cells (EPCs) and dental c

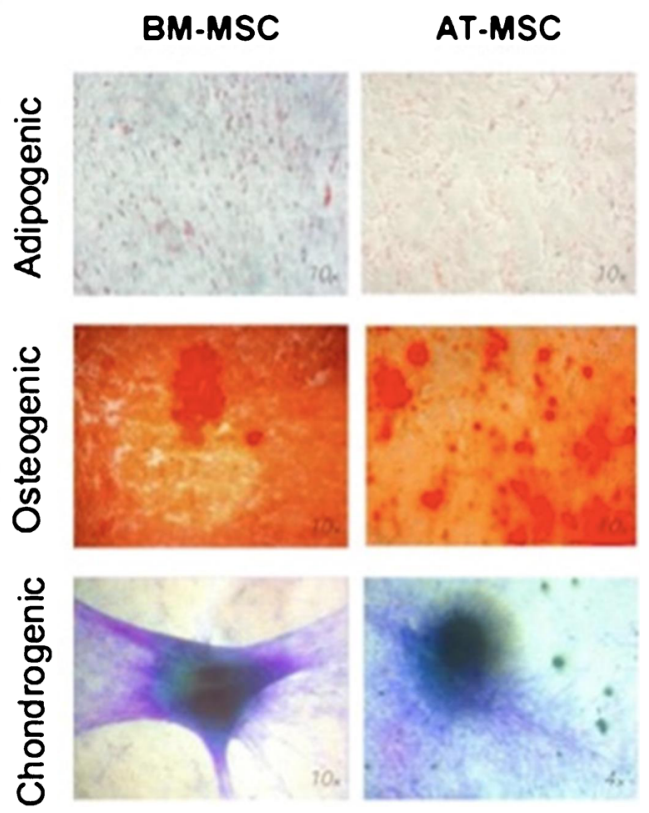

marrow, and adipose-derived MSCs exhibit strong differentiation potential toward osteogenic (Alizarin Red S, calcium deposits) and chondrogenic (Toluidine Blue, glycosaminoglycans), but not adipogenic (Oil Red O, lipid accumulation). Figure 1a reprinted from Amable et al. [40••]. Figures 1b, c reprinted from Kim et al. [48]

pulp stem cells, PRP was shown to increase VEGF and PDGF secretion thereby promoting vasculogenesis and stimulating EPCs to form vessel-like structures [59]. PRP contains high levels of angiopoietin 1 (Ang1), which mediates angiogenesis. Inhibition of Ang1-Tie2 signaling suppressed the proangiogenic effect of PRP [60]. Interestingly, PRP prevented endotoxin-induced pulmonary edema through the same pathway [61], which might be due to the PRP's potential to stabilize vascular integrity and permeability. PRP diminished the disruption of cell-cell junctional integrity induced by inflammatory cytokines [61]. Indeed, PRP supplementation has consistently been associated with improved angiogenesis in a variety of models and methods of application (Fig. 3) [60, 62-64]. Therefore, blood derivatives such as PRP could be explored as an autologous competitor for the traditionally used recombinant protein VEGF to induce blood vessel formation within implanted bioengineered constructs.

\section{Chemotaxis and Inflammation}

In addition to PRP's and PRF's direct effects on proliferation, differentiation, and angiogenesis, they also affect wound healing indirectly via the chemotactic recruitment of cells and local control of the inflammatory environment. Indeed, PRP has been reported to chemotactically attract human MSCs [54]. Another promising chemotactic effect was also observed in a rat tendon healing model, where PRP was able 
Fig. 3 Angiogenic effect of PRP on human dermal microvascular endothelial cells. a In vitro tube formation assay and $\mathbf{b}$ mean tube length showing PRP's proangiogenic effects. Reprinted from Mammoto et al. [60] a

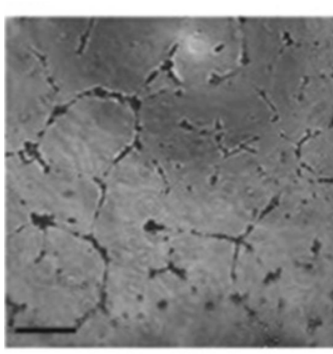

Control

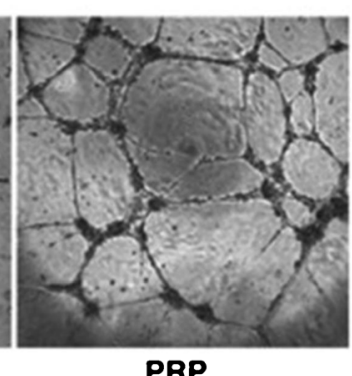

PRP b

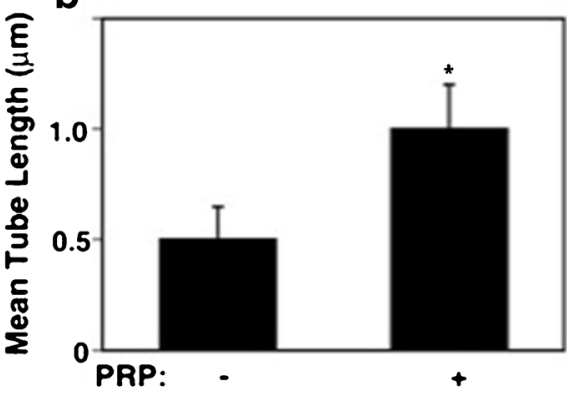

to recruit circulation-derived cells and help the initial stages of tendon healing [65]. In addition, it has been shown that PRP can attract peripheral blood monocytes in a dose-dependent manner, which also led to changes in the monocyte's proinflammatory cytokine release profile [66].

Multiple groups have reported on the capability PRP to mitigate inflammation. Activated PRP demonstrated high levels of hepatocyte growth factor (HGF), IL-4, and TNF $\alpha$ [67]. In chondrocytes, high levels of PRP-derived HGF and TNF- $\alpha$ decreased the trans-activating activity of NF- $\mathrm{KB}$ by acting as an anti-inflammatory trigger [67]. Additionally, in osteoarthritic chondrocytes challenged with IL1B, PRP reduced the levels of NF-KB activation and had multiple antiinflammatory effects [68]. In another osteoarthritic model utilizing osteoarthritic cartilage and synovium from patients, PRP, with or without leukocytes, had similar antiinflammatory effects [69]. Moreover, in tendon cells treated with IL-1B, PRP induced the expression of VEGF, RANTES, and HGF and decreased pro-inflammatory cytokines IL-6, IL8 , and MCP-1 [70].

Taken together, the set of reports mentioned above highlight a role of PRP in growth factor release and its importance for chemotaxis and inflammation. Not surprisingly, PRP has been considered for the management of inflammation and pain for specific diseases such as osteoarthritis [71•].

\section{Neuroprotection and Other Effects}

In organ cocultures of brain cortex and spinal cord, PRP was also found to promote axon growth, mediated by IGF-1 and VEGF, but not by PDGF-AB [72]. Furthermore, inhibition of TGF $\beta-1$ results in higher axon growth, suggesting that TGF $\beta-1$ inhibits or impairs axon growth. Besides axon growth, PRP has neuroprotective properties. In a rat brain focal ischemic stroke model, PRP lysate administered systemically or locally provided neuroprotective effects [73]. However, the effects in reducing infarct volume were higher with local intra-arterial infusion of PRP lysate compared to systemic administration. Additionally, PRP minimized neurological deficits in the same model.

PRP also showed antimicrobial activity by inhibiting bacterial growth. Agar plates with PRP inhibited initial bacterial growth of Pseudomonas aeruginosa, Staphylococcus aureus, and Streptococcus faecalis [74]. This inhibition was mediated by the release of anti-bacterial chemokine ligand 1, 3, and 5 .

Interestingly, PRP was shown to upregulate the pluripotency markers and decrease lineage-specific marker expression in human MSCs, human muscle-derived progenitor cells, and adipose-derived stem cells [43, 75]. Moreover, PRP has been demonstrated to protect cell viability during cryopreservation [42]. Thus, PRP holds promise to augment stem cell expansion while minimizing loss of stemness.

\section{Clinical Translation}

PRP and PRF have many clinical applications in regenerative medicine. Numerous in vitro and animal studies consistently reported that these blood-derived products can promote stem cell differentiation to some cell lineages. Currently, stem cell laden PRP scaffolds are clinically explored. In particular, they are investigated in maxillofacial procedures, orthopedic surgeries, and wound or burn therapies.

PRP has been reported to accelerate chronic skin ulcer repair as well as improve fat graft outcomes. For instance, patients with lower extremity ulcers were treated with PRP mixed by fat tissue base or with a control treatment consisting of collagen mixed with hyaluronic acid. After 7 weeks and 10 weeks, the wounds treated with PRP were reepithelialized completely in respectively 61.1 and $88.9 \%$, compared to 40 and $60 \%$ of control group. A similar study was held for to explore the effect of PRP on the treatment of different maxillofacial defects. Patients received either treatment with PRP and fat grafting or fat graft only. One year post surgery, graft survival, and maintenance of three-dimensional tissue volume were $70 \%$ in PRP-treated group, as compared to $31 \%$ in the fat graft only control group [76].

In the orthopedic field PRP is regularly included as a bioactive component. For example, in a human clinical trial to treat deformities, 21 patients underwent high tibial osteotomy and MSC therapy in conjunction with PRP injection, while 23 control patients received high tibial osteotomy with PRP injection only. This demonstrated that the addition of MSCs significantly reduced both pain and increased (fibro)cartilage formation (Fig. 4) [77•]. Similarly, Koh et al. treated patients 
Fig. 4 Arthroscopic intraarticular view of an osteoarthritic knee a before treatment demonstrating cartilage defects on the articular surface and $\mathbf{b}$ 17 months posttreatment with MSC and PRP, which demonstrates significant cartilage regeneration on defect site. Reprinted from Koh et al. [77•]
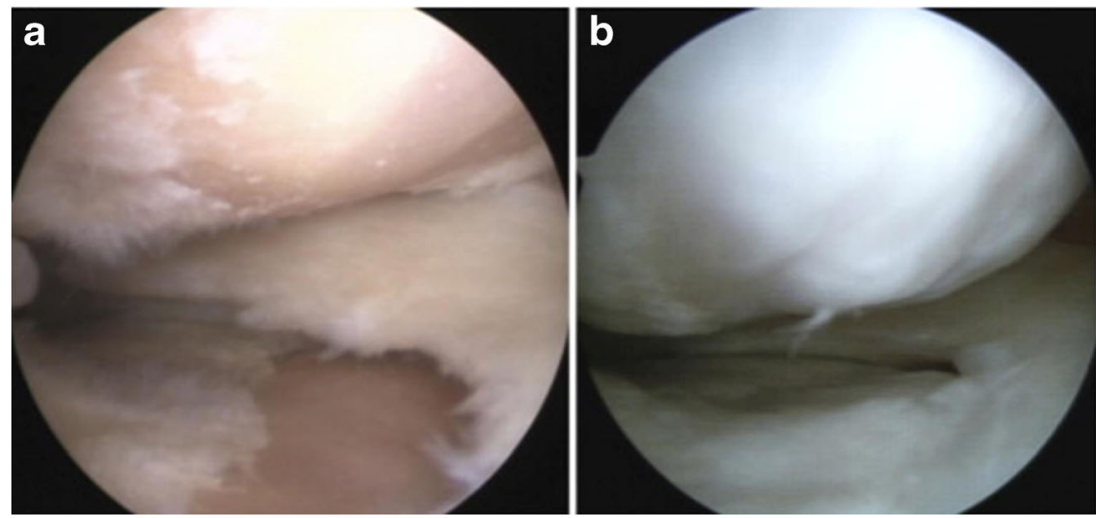

with osteoarthritic knees using a combination of MSCs, isolated from the infrapatellar fat pad, and with PRP, while control patients received PRP injection without stem cell supplementation. The mixture was injected into osteoarthritic knees once a week for up to 3 weeks [78]. Short-term results suggested that MSC supplementation reduced pain and improved joint function in treated patients. Although these particular studies explored the potential healing effect of MSCs, the clinical effects of PRP have remained to be fully investigated. Regardless, they were able to confirm provide proof-ofprinciple for the safety of the clinical use of PRP.

\section{a}

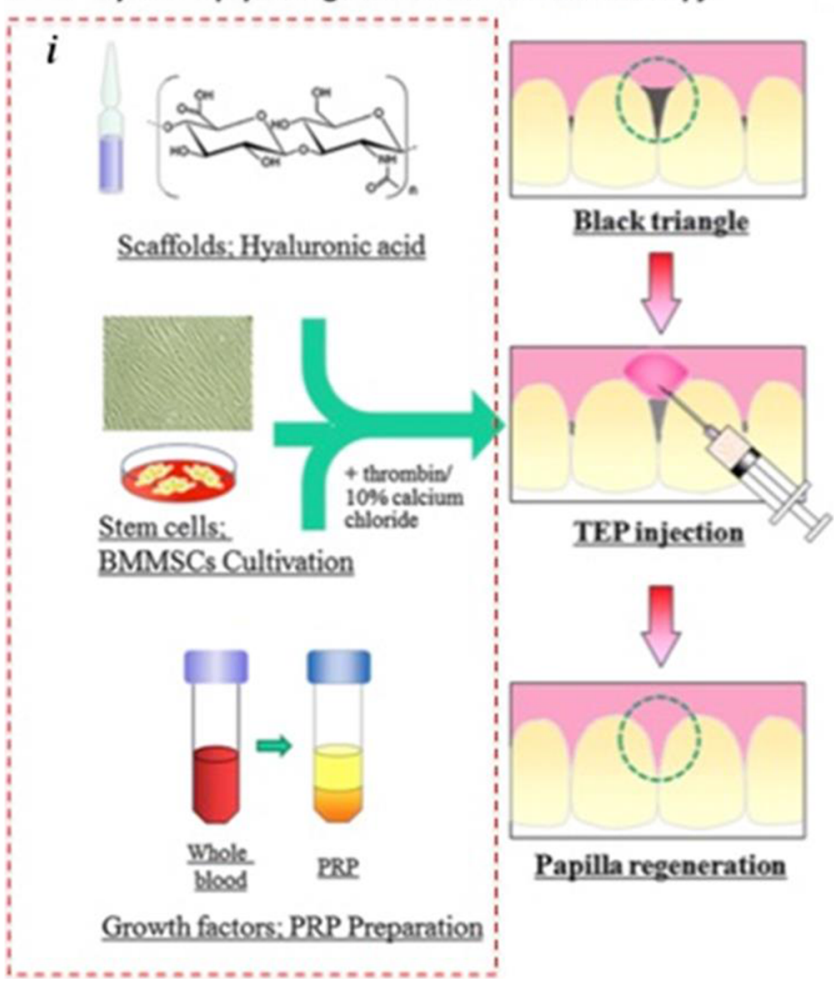

Fig. 5 Application of MSCs, PRP, and hyaluronic acid in papilla regeneration in patients with Black Triangle defect. a Schematic depiction of the preparation and injection procedure. $\mathbf{b}$ Photographs that
In the maxillofacial/dentistry field, PRP was explored to treat a Black Triangle, which is the interproximal distance between the teeth caused by receded papilla height. Ten patients received between one and five of injections and were followed up for period up to 69 months. All patients reported high satisfaction with cosmetic results without any major adverse effects. Patients demonstrated various degrees of regeneration and defect filling, with two patients demonstrating full regeneration (Fig. 5) [79]. However, the involvement of progenitor cells in this regeneration remained largely uninvestigated.

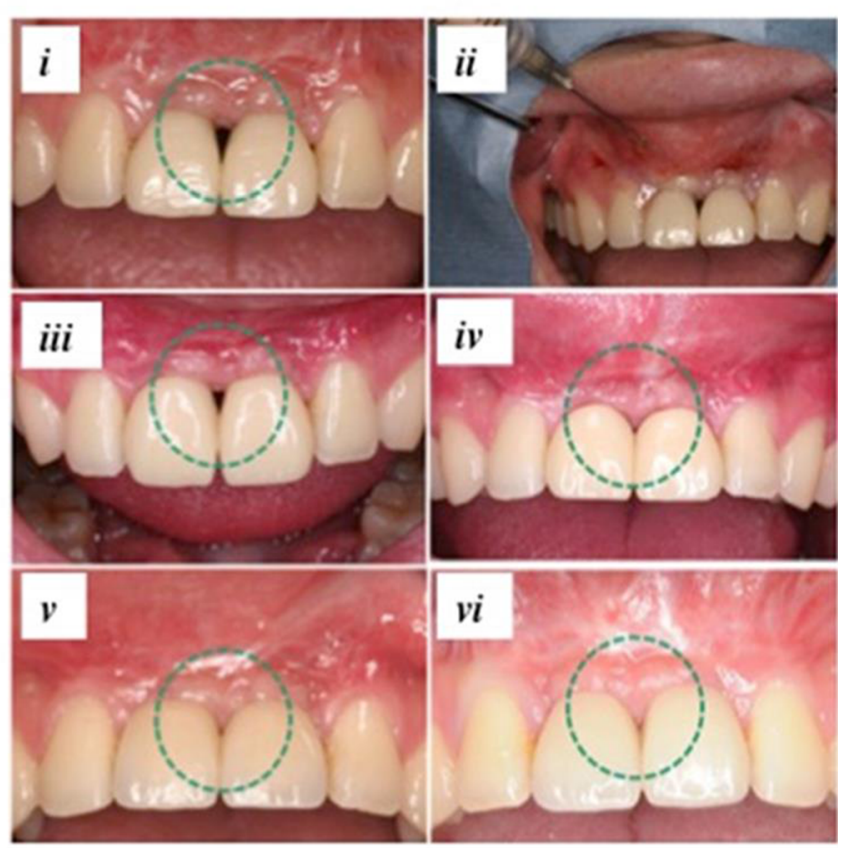

visualize the defect's healing during (i) pretreatment, (ii) treatment, (iii) 3, (iv) 6, (v) 12, and (vi) 36 months post-treatment. Reprinted from Yamada et al. [79] 
In summary, the early clinical results indicated that plateletbased blood derivatives such as PRP and potentially PRF are promising supplements for current stem cell-based therapies. In particular, they have consistently been shown to represent a safe and readily available source of growth factors.

\section{Future Challenges}

Although several reports have elaborated on the advantages of blood derivative in the fields of orthopedics, dermatology, and vascular applications, there are still several challenges to be addressed.

The composition of blood derivatives displays relatively strong intra-patient variation [51]. Moreover, compositional variation is correlated with age, sex, and patient comorbidities. Furthermore, it remains largely unstudied how these changes will affect stem cell behavior in vivo. Better characterization of PRP and PRF proteomes is therefore highly desired to enable more robust and predictable clinical outcomes. In addition, to the intra-patient variation, previous reports have underlined that the composition of each patient is also affected by the subsequent preparation methodology [80, 81]. Unfortunately, currently, there is no universally accepted standardized protocol. These incongruences reduce the comparability and reproducibility of the various PRP studies. These challenges can be addressed by initiating systematic high throughput analyses. Alternatively, it might be argued that the generation of multiple donor pools might mitigate this challenge, but this approach will come at the cost of its otherwise autologous nature.

Besides the blood derivative's composition, its delivery will potently affect its effect on stem cells and tissue regeneration. In particular, biomaterial characteristics such as stiffness, degradability, porosity, and bioactivity are likely to influence the blood derivative's effect on stem cells and tissue regeneration. Although several studies started to explore the concept of combining PRP and PRF with biomaterials, comprehensive or systematic approaches have not been performed. Furthermore, biomaterials can be leveraged to overcome the challenge of prolonging PRP and PRF delivery. Currently, their delivery relies on poorly controlled bulk release. In consequence, prolonged treatments require multiple treatments e.g. numerous injections. This results in strongly fluctuating growth factor concentrations, which impairs clinical predictability. Biomaterials can act as controlled release devices, which will allow for sustained or even ondemand delivery of these growth factor cocktails. In addition, it can be envisioned that biomaterials can covalently bind specific growth factors to locally retain high levels of these molecules.

\section{Conclusion}

Blood derivatives, and in particular PRP, represent a promising source of natural autologous growth factors that potentiate stem cell proliferation, migration, and differentiation. Moreover, clinical trials have confirmed PRP's safety as well as its ability to improve the clinical outcomes of stem cellbased therapies. However, gaining control over blood derivatives, chemical composition remains a key challenge to achieve reproducible and predictable clinical results.

Acknowledgments The authors acknowledge funding from the National Science Foundation (EFRI-1240443), IMMODGEL (602694), and the National Institutes of Health (EB012597, AR057837, DE021468, HL099073, AI105024, AR063745). Dr. Leijten acknowledges financial support from Innovative Research Incentives Scheme Veni \#14328 of the Netherlands Organization for Scientific Research (NWO). J.R. acknowledges the support from the Portuguese Foundation for Science and Technology (FCT; SFRH/BD/51679/2011).

\section{Compliance with Ethical Standards}

Conflict of Interest Elham Masoudi, João Ribas, Gaurav Kaushik, Jeroen Leijten, and Ali Khademhosseini declare that they have no conflict of interest.

Human and Animal Rights and Informed Consent This article does not contain any studies with human or animal subjects performed by any of the authors.

\section{References}

Papers of particular interest, published recently, have been highlighted as:

- Of importance

•-Of major importance

1. Langer R, Vacanti JP. Tissue engineering. Science. 1993;260:920 6.

2. Leijten J, Chai YC, Papantoniou I, et al. Cell based advanced therapeutic medicinal products for bone repair: keep it simple? Adv Drug Deliv Rev. 2015;84:30-44.

3. Anitua E, Tejero R, Alkhraisat MH, et al. Platelet-rich plasma to improve the bio-functionality of biomaterials. BioDrugs. 2013;27: 97-111.

4. De Pascale MR, Sommese L, Casamassimi A, et al. Platelet derivatives in regenerative medicine: an update. Transfus Med Rev. 2015;29:52-61.

5. Sell SA, Wolfe PS, Ericksen JJ, et al. Incorporating platelet-rich plasma into electrospun scaffolds for tissue engineering applications. Tissue Eng A. 2011;17:2723-37.

6. Liao H-T, Marra KG, Rubin JP. Application of platelet-rich plasma and platelet-rich fibrin in fat grafting: basic science and literature review. Tissue Eng B Rev. 2014;20:267-76.

7. Sánchez-González DJ, Méndez-Bolaina E, Trejo-Bahena NI. Platelet-rich plasma peptides: key for regeneration. Int J Peptides. 2012;2012:532519.

8.• Amable PR, Carias RBV, Teixeira MVT, et al. Platelet-rich plasma preparation for regenerative medicine: optimization and 
quantification of cytokines and growth factors. Stem Cell Res Ther. 2013;4:67. This study explores the effects of different variables such as time and temperature on PRP's chemical composition.

9. Dhurat R, Sukesh M. Principles and methods of preparation of platelet-rich plasma: a review and author's perspective. J Cutaneous Aesthetic Surg. 2014;7:189-97.

10. Dohan Ehrenfest DM, Rasmusson L, Albrektsson T. Classification of platelet concentrates: from pure platelet-rich plasma (P-PRP) to leucocyte- and platelet-rich fibrin (L-PRF). Trends Biotechnol. 2009;27:158-67.

11. Zhong W, Sumita $\mathrm{Y}$, Ohba S, et al. In vivo comparison of the bone regeneration capability of human bone marrow concentrates vs. platelet-rich plasma. PLoS One. 2012;7:e40833.

12. Perez AGM, Lana JFSD, Rodrigues AA, et al. Relevant aspects of centrifugation step in the preparation of platelet-rich plasma. ISRN Hematol. 2014;2014:176060.

13. Schär MO, Diaz-Romero J, Kohl S, et al. Platelet-rich concentrates differentially release growth factors and induce cell migration in vitro. Clin Orthop Relat Res. 2015;473:1635-43.

14. Snyder EL, Calhoun BC. Topical platelet growth factor therapy: of lotions and potions. Transfusion. 2001;41:1186-9.

15. Anilkumar K, Geetha A, Umasudhakar, et al. Platelet-rich-fibrin: a novel root coverage approach. J Indian Soc Periodontol. 2009;13: $50-4$.

16. Ghanaati S, Booms P, Orlowska A, et al. Advanced platelet-rich fibrin: a new concept for cell-based tissue engineering by means of inflammatory cells. J Oral Implantol. 2014;40:679-89.

17. Christoffersson G, Vågesjö E, Vandooren J, et al. VEGF-A recruits a proangiogenic MMP-9 - delivering neutrophil subset that induces angiogenesis in transplanted hypoxic tissue. Blood. 2012;120: 4653-62.

18. Wang M, Li J, Liu J, et al. [The comparison of platelet-rich fibrin and platelet-rich plasma in releasing of growth factors and their effects on the proliferation and differentiation of adipose tissuederived stem cells in vitro]. Hua xi kou qiang yi xue za zhi $=$ Huaxi kouqiang yixue zazhi $=$ West China journal of stomatology 2012;30:641-4, 9 .

19. He L, Lin $\mathrm{Y}, \mathrm{Hu} \mathrm{X}$, et al. A comparative study of platelet-rich fibrin (PRF) and platelet-rich plasma (PRP) on the effect of proliferation and differentiation of rat osteoblasts in vitro. Oral Surg, Oral Med, Oral Pathol, Oral Radiol Endodontol. 2009;108:707-13.

20. Ehrenfest DMD, Peppo GMd, Doglioli P, et al. Slow release of growth factors and thrombospondin-1 in Choukroun's platelet-rich fibrin (PRF): a gold standard to achieve for all surgical platelet concentrates technologies. Growth Factors 2009.

21. Su CY, Kuo YP, Tseng YH, et al. In vitro release of growth factors from platelet-rich fibrin (PRF): a proposal to optimize the clinical applications of PRF. Oral Surg, Oral Med, Oral Pathol, Oral radiol Endodontics. 2009;108:56-61.

22. Choukroun J. Advanced PRF, \& i-PRF: platelet concentrates or blood concentrates? J Periodontal Med Clin Pract. 2014;1:3.

23. Dohan DM, Choukroun J, Diss A, et al. Platelet-rich fibrin (PRF): a second-generation platelet concentrate. Part III: leucocyte activation: a new feature for platelet concentrates? Oral Surgery, Oral Med, Oral Pathol, Oral Radiol Endodontol. 2006;101:e51-5.

24. Dohan DM, Choukroun J, Diss A, et al. Platelet-rich fibrin (PRF): a second-generation platelet concentrate. Part III: leucocyte activation: a new feature for platelet concentrates? Oral Surg, Oral Med, Oral Pathol, Oral Radiol Endodontics. 2006;101:e51-5.

25. Lu HH, Vo JM, Chin HS, et al. Controlled delivery of platelet-rich plasma-derived growth factors for bone formation. J Biomedical Mater Res Part A. 2008;86:1128-36.

26. Celotti F, Colciago A, Negri-Cesi P, et al. Effect of platelet-rich plasma on migration and proliferation of SaOS-2 osteoblasts: role of platelet-derived growth factor and transforming growth factor- beta. Wound Repair Regen: Off Publ Wound Healing Soc Eur Tissue Repair Soc. 2006;14:195-202.

27. Yang HS, Shin J, Bhang SH, et al. Enhanced skin wound healing by a sustained release of growth factors contained in platelet-rich plasma. Exp Mol Med. 2011;43:622-9.

28. Akeda K, An HS, Okuma M, et al. Platelet-rich plasma stimulates porcine articular chondrocyte proliferation and matrix biosynthesis. Osteoarthritis Cartil / OARS, Osteoarthritis Res Soc. 2006;14: 1272-80.

29. Arpornmaeklong P, Kochel M, Depprich R, et al. Influence of platelet-rich plasma (PRP) on osteogenic differentiation of rat bone marrow stromal cells. An in vitro study. Int J Oral Maxillofac Surg. 2004;33:60-70.

30. Choi BH, Zhu SJ, Kim BY, et al. Effect of platelet-rich plasma (PRP) concentration on the viability and proliferation of alveolar bone cells: an in vitro study. Int J Oral Maxillofac Surg. 2005;34: $420-4$.

31. Creeper F, Lichanska AM, Marshall RI, et al. The effect of plateletrich plasma on osteoblast and periodontal ligament cell migration, proliferation and differentiation. J Periodontal Res. 2009;44:25865.

32. Drengk A, Zapf A, Sturmer EK, et al. Influence of platelet-rich plasma on chondrogenic differentiation and proliferation of chondrocytes and mesenchymal stem cells. Cells Tissues Organs. 2009;189:317-26.

33. Frechette JP, Martineau I, Gagnon G. Platelet-rich plasmas: growth factor content and roles in wound healing. J Dent Res. 2005;84 434-9.

34. Kanno T, Takahashi T, Tsujisawa T, et al. Platelet-rich plasma enhances human osteoblast-like cell proliferation and differentiation. J Oral Maxillofacial Surg. 2005;63:362-9.

35. Han J, Meng HX, Tang JM, et al. The effect of different platelet-rich plasma concentrations on proliferation and differentiation of human periodontal ligament cells in vitro. Cell Prolif. 2007;40:241-52.

36. Kawase T, Okuda K, Saito Y, et al. In vitro evidence that the biological effects of platelet-rich plasma on periodontal ligament cells is not mediated solely by constituent transforming-growth factorbeta or platelet-derived growth factor. J Periodontol. 2005;76:760 7.

37. Jo CH, Kim JE, Yoon KS, et al. Platelet-rich plasma stimulates cell proliferation and enhances matrix gene expression and synthesis in tenocytes from human rotator cuff tendons with degenerative tears. Am J Sports Med. 2012;40:1035-45.

38. Yu TY, Pang JH, Wu KP, et al. Platelet-rich plasma increases proliferation of tendon cells by modulating Stat3 and p27 to upregulate expression of cyclins and cyclin-dependent kinases. Cell Prolif. 2015;48:413-20.

39. Kakudo N, Morimoto N, Kushida S, et al. Platelet-rich plasma releasate promotes angiogenesis in vitro and in vivo. Med Mol Morphol. 2014;47:83-9.

40.• Amable PR, Teixeira MV, Carias RB, et al. Mesenchymal stromal cell proliferation, gene expression and protein production in human platelet-rich plasma-supplemented media. PLoS One. 2014;9: e104662. This study provides valuable insights into the effect of PRP on human mesenchymal stromal cells derived from different tissues, with detailed analysis on secreted proteins.

41. Mishra A, Tummala P, King A, et al. Buffered platelet-rich plasma enhances mesenchymal stem cell proliferation and chondrogenic differentiation. Tissue Eng Part C, Methods. 2009;15:431-5.

42. Murphy MB, Blashki D, Buchanan RM, et al. Adult and umbilical cord blood-derived platelet-rich plasma for mesenchymal stem cell proliferation, chemotaxis, and cryo-preservation. Biomaterials. 2012;33:5308-16.

43. Roubelakis MG, Trohatou O, Roubelakis A, et al. Platelet-rich plasma (PRP) promotes fetal mesenchymal stem/stromal cell 
migration and wound healing process. Stem Cell Rev. 2014;10: 417-28.

44. D'Esposito V, Passaretti F, Perruolo G, et al. Platelet-Rich Plasma Increases Growth and Motility of Adipose Tissue-Derived Mesenchymal Stem Cells and Controls Adipocyte Secretory Function. J Cell Biochem. 2015;116:2408-18.

45. Diaz-Gomez L, Alvarez-Lorenzo C, Concheiro A, et al. Biodegradable electrospun nanofibers coated with platelet-rich plasma for cell adhesion and proliferation. Mater Sci Eng C, Mater Biol Appl. 2014;40:180-8.

46. Fukaya Y, Kuroda M, Aoyagi Y, et al. Platelet-rich plasma inhibits the apoptosis of highly adipogenic homogeneous preadipocytes in an in vitro culture system. Exp Mol Med. 2012;44:330-9.

47. Xu FT, Li HM, Yin QS, et al. Effect of activated autologous platelet-rich plasma on proliferation and osteogenic differentiation of human adipose-derived stem cells in vitro. Am J Transl Res. 2015;7:257-70.

48. Kim YH, Furuya H, Tabata Y. Enhancement of bone regeneration by dual release of a macrophage recruitment agent and platelet-rich plasma from gelatin hydrogels. Biomaterials. 2014;35:214-24.

49. Gandhi A, Doumas C, O'Connor JP, et al. The effects of local platelet rich plasma delivery on diabetic fracture healing. Bone. 2006;38:540-6.

50. Sarkar MR, Augat P, Shefelbine SJ, et al. Bone formation in a long bone defect model using a platelet-rich plasma-loaded collagen scaffold. Biomaterials. 2006;27:1817-23.

51. Eppley BL, Woodell JE, Higgins J. Platelet quantification and growth factor analysis from platelet-rich plasma: implications for wound healing. Plast Reconstr Surg. 2004;114:1502-8.

52. Zhang J, Wang JH. Platelet-rich plasma releasate promotes differentiation of tendon stem cells into active tenocytes. Am J Sports Med. 2010;38:2477-86.

53. de Boer HC, Verseyden C, Ulfman LH, et al. Fibrin and activated platelets cooperatively guide stem cells to a vascular injury and promote differentiation towards an endothelial cell phenotype. Arterioscler Thromb Vasc Biol. 2006;26:1653-9.

54. Moreira Teixeira LS, Leijten JC, Wennink JW, et al. The effect of platelet lysate supplementation of a dextran-based hydrogel on cartilage formation. Biomaterials. 2012;33:3651-61.

55. Tomoyasu A, Higashio K, Kanomata K, et al. Platelet-rich plasma stimulates osteoblastic differentiation in the presence of BMPs. Biochem Biophys Res Commun. 2007;361:62-7.

56. Kruger JP, Hondke S, Endres M, et al. Human platelet-rich plasma stimulates migration and chondrogenic differentiation of human subchondral progenitor cells. J Orthopaedic Res. 2012;30:845-52.

57. do Amaral RJ, Matsiko A, Tomazette MR, et al. Platelet-rich plasma releasate differently stimulates cellular commitment toward the chondrogenic lineage according to concentration. J Tissue Eng. 2015;6:2041731415594127.

58. Kruger JP, Ketzmar AK, Endres M, et al. Human platelet-rich plasma induces chondrogenic differentiation of subchondral progenitor cells in polyglycolic acid-hyaluronan scaffolds. J Biomed Mater Res B Appl Biomater. 2014;102:681-92.

59. Li X, Hou J, Wu B, et al. Effects of platelet-rich plasma and cell coculture on angiogenesis in human dental pulp stem cells and endothelial progenitor cells. J Endod. 2014;40:1810-4.

60. Mammoto T, Jiang A, Jiang E, et al. Platelet rich plasma extract promotes angiogenesis through the angiopoietin1-Tie2 pathway. Microvasc Res. 2013;89:15-24.

61. Mammoto T, Jiang A, Jiang E, et al. Platelet-rich plasma extract prevents pulmonary edema through angiopoietin-Tie2 signaling. Am J Respir Cell Mol Biol. 2015;52:56-64.

62. Kakudo N, Kushida S, Minakata T, et al. Platelet-rich plasma promotes epithelialization and angiogenesis in a splitthickness skin graft donor site. Med Mol Morphol. 2011;44:233-6.
63. Notodihardjo PV, Morimoto N, Kakudo N, et al. Gelatin hydrogel impregnated with platelet-rich plasma releasate promotes angiogenesis and wound healing in murine model. J Artificial Organs. 2015;18:64-71.

64. Roy S, Driggs J, Elgharably H, et al. Platelet-rich fibrin matrix improves wound angiogenesis via inducing endothelial cell proliferation. Wound Repair Regen. 2011;19:753-66.

65. Kajikawa Y, Morihara T, Sakamoto H, et al. Platelet-rich plasma enhances the initial mobilization of circulation-derived cells for tendon healing. J Cell Physiol. 2008;215:837-45.

66. El-Sharkawy H, Kantarci A, Deady J, et al. Platelet-rich plasma: growth factors and pro- and anti-inflammatory properties. J Periodontol. 2007;78:661-9.

67. Bendinelli P, Matteucci E, Dogliotti G, et al. Molecular basis of anti-inflammatory action of platelet-rich plasma on human chondrocytes: mechanisms of NF-kappaB inhibition via HGF. J Cell Physiol. 2010;225:757-66.

68. van Buul GM, Koevoet WL, Kops N, et al. Platelet-rich plasma releasate inhibits inflammatory processes in osteoarthritic chondrocytes. Am J Sports Med. 2011;39:2362-70.

69. Osterman C, McCarthy MB, Cote MP, et al. Platelet-Rich Plasma Increases Anti-inflammatory Markers in a Human Coculture Model for Osteoarthritis. Am J Sports Med. 2015;43:1474-84.

70. Andia I, Rubio-Azpeitia E, Maffulli N. Platelet-rich plasma modulates the secretion of inflammatory/angiogenic proteins by inflamed tenocytes. Clin Orthop Relat Res. 2015;473:1624-34.

71. Andia I, Maffulli N. Platelet-rich plasma for managing pain and inflammation in osteoarthritis. Nat Rev Rheumatol. 2013;9:72130. This review provides an elegant overview of the usage of PRP for inflammation and osteoarthritis.

72. Takeuchi M, Kamei N, Shinomiya R, et al. Human platelet-rich plasma promotes axon growth in brain-spinal cord coculture. Neuroreport. 2012;23:712-6.

73. Zhang Y, Ying G, Ren C, et al. Administration of human plateletrich plasma reduces infarction volume and improves motor function in adult rats with focal ischemic stroke. Brain Res. 2015;1594:26773.

74. Mariani E, Filardo G, Canella V, et al. Platelet-rich plasma affects bacterial growth in vitro. Cytotherapy. 2014;16:1294-304.

75. Li H, Usas A, Poddar M, et al. Platelet-rich plasma promotes the proliferation of human muscle derived progenitor cells and maintains their stemness. PLoS One. 2013;8:e64923.

76. Cervelli V, Gentile P, Scioli MG, et al. Application of platelet-rich plasma in plastic surgery: clinical and in vitro evaluation. Tissue Eng Part C, Methods. 2009; 15:625-34.

77. Koh Y-G, Kwon O-R, Kim Y-S, et al. Comparative outcomes of open-wedge high tibial osteotomy with platelet-rich plasma alone or in combination with mesenchymal stem cell treatment: a prospective study. Arthroscopy. 2014;30:1453-60. This study demonstrated that pain relief and cartilage formation is significantly improved in patients treated treated with PRP and stem cells as compared to PRP alone.

78. Koh Y-G, Choi Y-J. Infrapatellar fat pad-derived mesenchymal stem cell therapy for knee osteoarthritis. Knee. 2012;19:902-7.

79. Yamada Y, Nakamura S, Ueda M, et al. Papilla regeneration by injectable stem cell therapy with regenerative medicine: long-term clinical prognosis. J Tissue Eng Regen Med. 2015;9:305-9.

80. Landesberg R, Roy M, Glickman RS. Quantification of growth factor levels using a simplified method of platelet-rich plasma gel preparation. J Oral Maxillofac Surg. 2000;58:297-300.

81. Leitner GC, Gruber R, Neumuller J, et al. Platelet content and growth factor release in platelet-rich plasma: a comparison of four different systems. Vox Sang. 2006;91:135-9. 